\title{
Molecular tilting and columnar stacking of Fe phthalocyanine thin films on $\mathrm{Au}(111)$
}

\author{
F. Bartolomé, ${ }^{1, a)}$ O. Bunău, ${ }^{1}$ L. M. García, ${ }^{1}$ C. R. Natolii, ${ }^{2}$ M. Piantek, ${ }^{1,3}$ J. I. Pascual,,${ }^{3,4}$ \\ I. K. Schuller, ${ }^{5}$ T. Gredig, ${ }^{6}$ F. Wilhelm, ${ }^{7}$ A. Rogalev, ${ }^{7}$ and J. Bartolomé ${ }^{1}$ \\ ${ }^{1}$ ICMA - Departamento de Física de la Materia Condensada, CSIC - Universidad de Zaragoza, \\ 50009 Zaragoza, Spain \\ ${ }^{2}$ INFN Laboratori Nazionali di Frascati, c.p. 13, I-00044 Frascati, Italy \\ ${ }^{3}$ Laboratorio de Microscopías Avanzadas, Instituto de Nanociencia de Aragón, University of Zaraqoza, \\ E-50018 Zaragoza, Spain \\ ${ }^{4}$ CIC nanoGUNE and Ikerbasque, Basque Foundation for Science, Tolosa Hiribidea 76, \\ 20018 San Sebastian-Donostia, Spain \\ ${ }^{5}$ Department of Physics and Center for Advanced Nanotechnology, University of California San Diego, \\ La Jolla, California 92093, USA \\ ${ }^{6}$ Department of Physics and Astronomy, California State University Long Beach, 1250 Bellflower Blvd., \\ Long Beach, California 90840-9505, USA \\ ${ }^{7}$ ESRF-The European Synchrotron, 38043 Grenoble Cedex 9, France
}

(Presented 5 November 2014; received 22 September 2014; accepted 15 November 2014; published online 30 March 2015)

Scanning tunneling microscopy and $\mathrm{x}$-ray absorption spectroscopic results at the $\mathrm{Fe} \mathrm{K}$ edge of $\mathrm{Fe}$ phthalocyanine $(\mathrm{FePc})$ thin films grown on $\mathrm{Au}$ substrates, together with theoretical calculations, allow us to refine the structure of the film. In particular, we show that the columnar stacking of the FePc molecules is different from that found in bulk $\alpha$ and $\beta$ phases. Moreover, the molecules do not lay parallel to the surface of the substrate. These structural findings are relevant to understand magnetism of FePc films. C 2015 AIP Publishing LLC. [http://dx.doi.org/10.1063/1.4916302]

Metallo-Phthalocyanines (MPc) are planar aromatic molecules with interesting physical properties which have played a role as model systems in basic science. MPc crystals form a herringbone-type columnar stacking structure. The columns are formed by molecules parallel to each other with central metal ions forming the axis of the column. FePc exhibits two monoclinic phases with a columnar stacking structure, differing in the tilt angle $\theta$ of the normal to the molecular plane with the stack axis: $\theta$ is $26.5^{\circ}$ for the $\alpha$ phase, while in the $\beta$ phase $\theta=44.8^{\circ}{ }^{1,2}$

The MPc molecules in powder bulk phase stack parallel to one another, so that the distance between molecules is approximately $d_{z} \approx 3.5 \pm 0.1 \AA$ in both $\alpha$ - and $\beta$-phases. In the $\alpha$-phase (see Fig. 1), the adjacent molecule is shifted by $d_{x y}= \pm 1.69 \AA$ along the $(1,1,0)$ axis, so that its $\mathrm{Fe}$ atom is near the center of one of the pyrrolic rings of the molecules above and below. ${ }^{1,2}$ In the $\beta$-phase (see Fig. 1), the shift is also along the $(1,1,0)$ direction but it amounts to $d_{x y}= \pm 3.38 \AA$, so that the Fe atom of the adjacent molecules is located directly above (or below) the $\mathrm{N}$ atom of a pyrrole ring.

The MPc ( $\mathrm{M}=3 \mathrm{~d}$ metal) films grown by organic molecular beam epitaxy (OMBE) technique ${ }^{3}$ have different crystalline orientations and order depending on the substrate type. ${ }^{4}$ The MPc molecular planes stack parallel to the substrate surface ("lying") when deposited on $\mathrm{Au}^{5,6}$ but inclined about $65^{\circ}$ ("standing") when deposited on sapphire ${ }^{3}$ or low work function metals like $\mathrm{Al}$ and $\mathrm{V}^{7}$ Besides, in a $\mathrm{CoPc}$ film grown on $\mathrm{Pb}$, a new staking was identified, with a shift of the molecules in adjacent layers of $d_{x y}= \pm 1.92 \AA$ in the $(1,0,0)$ direction (Fig. 1, thin film (TF)). ${ }^{8}$

\footnotetext{
a)Electronic mail: bartolom@unizar.es.
}

It was unexpected that bulk $\alpha$-phase FePc is ferromagnetic below $T_{C}=5 \mathrm{~K}$, while its $\beta$-phase remains paramagnetic down to the lowest temperatures. ${ }^{9,10}$ In a previous work on a FePc thin film, studied by means of X-ray magnetic circular dichroism at the $\mathrm{Fe} \mathrm{L}_{2,3}$ edges, it has been shown that the film has planar magnetic anisotropy, with a partially unquenched, large orbital moment. ${ }^{11}$ The origin of the ferromagnetism was associated to the special electronic state occupancy brought about by the rather high symmetry $\left(D_{4 h}\right)$ of the molecule (Fig. 1 top left), and the interactions with the neighboring molecules. Therefore, the determination of the detailed structure of FePc films is of high interest to understand its magnetism, as MPc deposited on a substrate are studied due to its possible applications in spintronics. FePc films deposited on Au show low temperature magnetic ordering and form a bidimensional crystal. ${ }^{11}$ Therefore, they offer the possibility of studying the fundamental electronic and magnetic properties in correlation with its stacking configuration. This work aims at solving the question on which

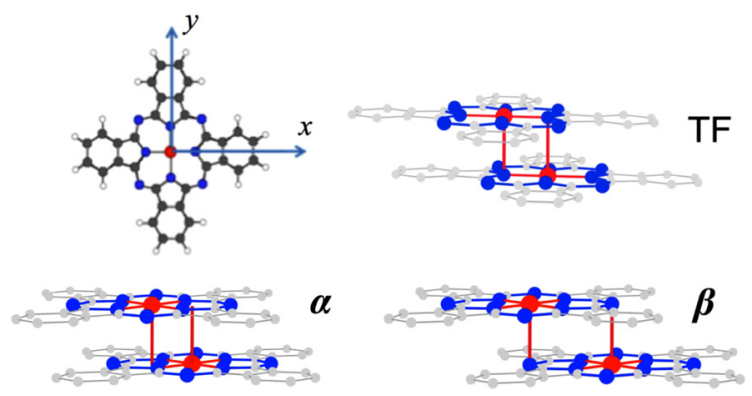

FIG. 1. (Top left) FePc molecule (Fe: red, N: blue, C: gray, and H: white). $\mathrm{TF}, \alpha$, and $\beta$ denote schemes of relative displacements of adjacent molecules within a columnar stack in the thin film (TF), $\alpha$-, and $\beta$ - configurations. 
is the actual stacking of the molecules of $\mathrm{FePc} / \mathrm{Au}$, whether $\alpha, \beta$, or TF, by means of a combined study of scanning tunneling microscopy (STM) and x-ray linearly polarized absorption (XLPA) spectroscopy.

STM measurements were performed at LMA, Zaragoza, on ultra-thin films of up to four monolayers of FePc evaporated on the (111) surface of an Au single crystal at RT. The metallic substrate was pretreated by cycles of subsequent $\mathrm{Ar}^{+}$sputtering and annealing at $600^{\circ} \mathrm{C}$. Molecules have been evaporated onto the atomically flat and clean substrate from an effusion cell at $330^{\circ} \mathrm{C}$. After preparation, the sample was introduced into a cryogenic STM operated at $4.6 \mathrm{~K}$ for the investigation of the organic film morphology.

The STM survey with bias voltage $V=+1.0 \mathrm{~V}$ of an FePc multilayer grown on $\mathrm{Au}(111)$ is shown in Fig. 2(a). At positive $V$, the unoccupied states of the sample are probed. Molecules are assembled in up to the fourth monolayer as indicated by the roman numerals in the figure. In the first layer (only a few molecules are visible in the survey picture), the molecules appear as cross-shapes features with their plane aligned parallel to the surface as typically observed for MPc on metallic substrates. ${ }^{4}$ The molecules assemble into a quadratic lattice following the three equivalent crystallographic axes of the $\mathrm{Au}(111) .{ }^{12}$ Here, the lattice parameter $a=13.0 \pm 0.2 \AA$ is slightly smaller than formerly reported $(15 \pm 1 \AA)$.

The quadratic unit cell is drawn on the overlying monolayers. However, there is a drastic change in topography with respect to the first monolayer. The molecules are no

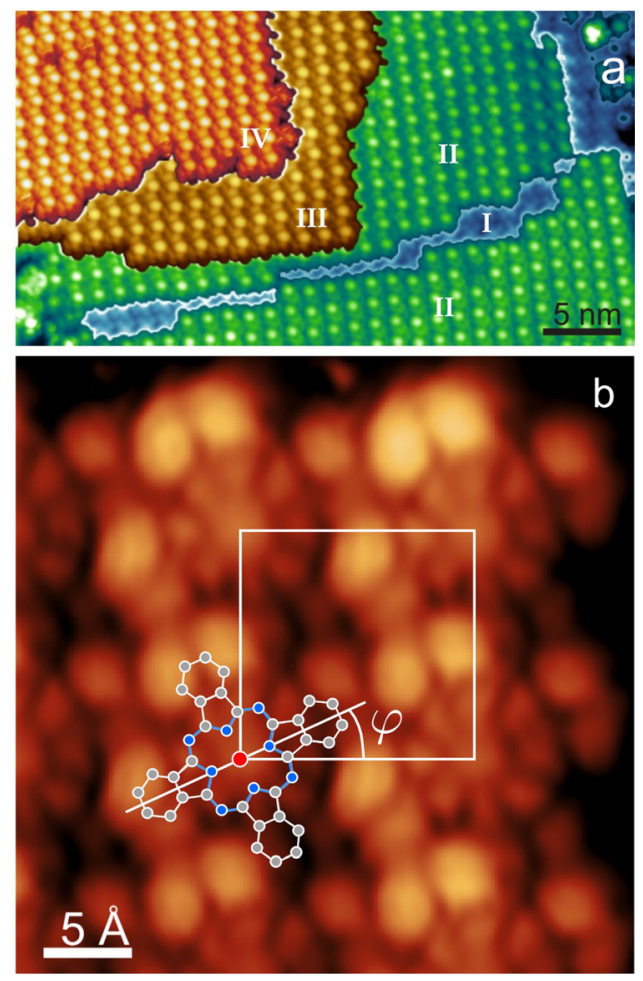

FIG. 2. (a) STM images $\left(\mathrm{V}_{\text {bias }}=1.0 \mathrm{~V}, \mathrm{I}=10 \mathrm{pA}\right)$ of FePc multilayer on $\mathrm{Au}(111)$. Roman numbers indicate the layer number. (b) Zoom into the 2nd layer at $\mathrm{V}_{\text {bias }}=-1.0 \mathrm{~V}$ and $\mathrm{I}_{T}=10 \mathrm{pA}$. Unit cell is indicated by the black rectangle. A molecule scheme is overlapped for clarity. longer visible as cross-shaped separated features. In the 2nd to 4th monolayers, they rather appear in identical regular intramolecular patterns of bright big protrusions (surrounded by smaller features). The change in the STM topography reveals a different electronic structure due to the decoupling from the metallic surface. The fact that there is no significant difference in topography for the three upper layers (II-IV) indicates that the gradual change of decoupling above the second monolayer is much smaller or even nonexistent.

A zoom into the second monolayer at negative $V=-1.0 \mathrm{~V}$ is given in Fig. 2(b). At $V<0$, we observe the occupied states and the STM topography resembles the Highest Occupied Molecular Orbital electronic density of states (DOS) in the corresponding voltage range, consisting of an inner ring of eight central lobes and eight additional lobes at the four outer phenyl rings. $V<0$ scans define better than $V>0$ ones the molecular alignment in the multilayer structure. The measured topography reflects this orbital distribution with an additional contribution at the Fe(II) center of the molecule which might come from the partial occupation of the metallic d-states in the multilayer.

The resolution of the sub-molecular electronic structure allows us to extract the molecular orientation in-plane with respect to the unit cell as well as out-of-plane with respect to the $\mathrm{Au}(111)$ surface. The former is given by the angle of $\varphi=26 \pm 1^{\circ}$ between the unit cell axis and the molecule's $\mathrm{Fe}-\mathrm{N}$ bond (see Fig. 2(b)). A tilt angle with respect to the $\mathrm{Au}(111)$ surface can be inferred from line scans of STM data as shown in Fig. 2(b). The analysis reveals a total tilt of the molecule $\left(\phi_{T}^{\mathrm{STM}} \sim 6 \pm 1^{\circ}\right)$ around the $(1,1,0)$ axis of the quadratic unit cell for all three overlayers. The drastic change of the molecules orientation for the second and higher layers is due to the dominant intermolecular interaction in the absence of the metallic surface.

Moreover, the relative position of the molecules in two adjacent layers can be distinguished by extrapolating the corresponding monolayer lattices. The shift of two adjacent layers runs along $\mathrm{Fe}-\mathrm{N}$ bond axis $(1,0,0)$ or conversely $(0,1,0)$, with a distance of $\pm d_{x y}=1.0 \pm 0.3 \AA$.

The FePc thin films used in the XLPA and X-ray natural linear dichroism (XNLD) were deposited on an atomically smooth gold substrate with the molecule plane lying flat on the substrate plane. ${ }^{13}$ Samples were fabricated and structurally characterized at UCSD. The thin film sample in this work was prepared by OMBE technique (details can be found in Refs. 3 and 14). In order to obtain FePc films with the molecule plane parallel to the sapphire substrate, first a $40 \mathrm{~nm}$ nominal thickness Au buffer layer was deposited at a rate of $0.3 \AA / \mathrm{s}$. This buffer layer was annealed for $1 \mathrm{~h}$ at $300^{\circ} \mathrm{C}$ in situ to remove water and reduce roughness at the Au surface. Without breaking vacuum, the FePc was deposited from a crucible set at $350^{\circ} \mathrm{C}$, while the substrate was maintained at $150^{\circ} \mathrm{C}$ to increase the crystallite size. The growth rate was $0.9 \AA / \mathrm{s}$. The FePc thickness of the investigated sample was $133 \mathrm{~nm}$.

$\mathrm{X}$-ray diffraction performed on this type of OMBE grown $\mathrm{FePc} / \mathrm{Au}$ has been shown that the Au buffer layer is constituted by crystals with $\mathrm{Au}(111)$ at the surface. The molecules are deposited in "lying" configuration patterns onto atomically 
smooth gold plated sapphire; ${ }^{15}$ the $z$ axis of the molecule being close to the normal to the substrate plane. Besides, the XLPA measurements performed at the $\mathrm{N}$ K-edge at normal $\left(\gamma=0^{\circ}\right)$ and grazing $\left(\gamma=75^{\circ}\right)$ incidence confirm that the molecules lie nearly parallel to the substrate. ${ }^{11}$

$\mathrm{X}$-ray absorption (XAS) near edge structure (XANES) and XLPA measurements at the Fe K edge on the FePc TF samples were performed at the ESRF ID12 beamline. The APPLE-II undulator and a double-Si-(111)-crystal monochromator were used to collect the spectra at the respective energies. The energy resolution around the Fe-K edge energy region is about $\Delta E / E=2 \times 10^{-4}$. All spectra were recorded by a fluorescence detector in backscattering geometry.

In Fig. 3, the normalized XLPA spectra measured at the Fe K-edge are shown. In normal mode $\left(\gamma=0^{\circ}\right)$, the electric field of the incoming beam $\mathbf{E}$ is parallel to the substrate and nearly to the molecule plane, while in grazing mode $\left(\gamma=75^{\circ}\right)$, the $\mathbf{E}$ forms an angle of $15^{\circ}$ with the normal to the molecule plane.

In a preliminary description, three spectral energy regions can be identified for the Fe K/edge: a pre-edge region, a rising edge, and a main absorption band. Prepeak(s) may occur corresponding to dipolar $1 \mathrm{~s} \rightarrow 4 \mathrm{p}$ transitions with $3 \mathrm{~d}-4 \mathrm{p}$ mixing and quadrupolar transitions $1 \mathrm{~s} \rightarrow 3 \mathrm{~d}$ (Fig. 3, peak A). In the present case, assuming that the stack contains an inversion center, as it is the case in the crystal, $3 \mathrm{~d}-4 \mathrm{p}$ hybridization of Fe states is forbidden by symmetry since these states belong to odd and even irreducible representations, respectively. Any non-zero contribution may, in principle, come from hybridization with ligand states or $3 \mathrm{~d}$ states from the surrounding n.n. molecules, caused by a slight breaking of the inversion symmetry. This contribution is negligible, as proven by FDMNES calculations, due to the large distance between Fe atoms in the stack. A second contribution may arise from quadrupolar $1 \mathrm{~s} \rightarrow 3 \mathrm{~d}$ excitations. The shoulder $\mathrm{B}$ and the main peak at $\mathrm{C}$ observed in normal incidence are assigned to $1 \mathrm{~s} \rightarrow 4 \mathrm{p}_{z}$ of Fe hybridized with $2 \mathrm{p}_{z}$ states of the $\mathrm{N}$ ligands of the molecules above and below. Other maxima (labelled D, E, F, G, and H) of the absorption

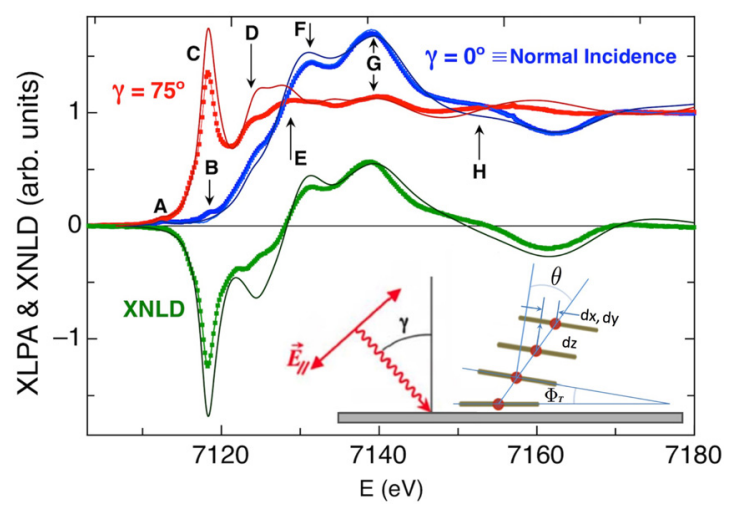

FIG. 3. Normalized XLPA and XNLD spectra of FePc film recorded at the Fe K-edge, at normal incidence (blue, $\gamma=0^{\circ}$ ), and grazing incidence (red, $\gamma=75^{\circ}$ ), at $T=300 \mathrm{~K}$. The inset shows a scheme of the incoming beam at incidence angle $\gamma$ with the electric field $\mathbf{E}$ in the horizontal $(\mathrm{H})$ polarization mode and a scheme of the stacking main parameters. FDMNES calculations are solid lines, experimental data in dots. curves in the main absorption band correspond to transitions to continuum states of p-character of the bottom of the conduction band and are very sensitive to the relative positions of the atoms in the molecular stack. XNLD, shown in Fig. 3, is obtained as: $\mu_{\mathrm{XNLD}}=\mu_{\|}-\mu_{\perp}$.

In XLPA measurements, the main peak $\mathrm{C}$ evolves from a maximum amplitude for grazing incidence (i.e., $\mathbf{E}$ out of the molecule plane) to nearly no peak for $\mathrm{N}$ mode ( $\mathbf{E}$ parallel to the molecule). The absorption is proportional to the number of empty valence states in the direction of the electric field $\mathbf{E}$. Therefore, this peak can be attributed to the excitations to the $\mathrm{p}_{\mathrm{z}}$-like molecular states mentioned above. Since in this sample there is a random distribution of molecules in the $x y$ plane, the spectra are averaged over the azimuthal angles, while all molecules have a nearly common $\mathrm{z}$ axis. As a consequence, in this effective $\mathrm{D}_{4 h}$ symmetry, the XLPA spectra as a function of incident angle $\gamma$ are related to the spectra $\mu_{\|}$ (polarization parallel to the substrate) and $\mu_{\perp}$ (polarization normal to the substrate) by $\mu(\gamma)=\mu_{\|} \cos ^{2} \gamma+\mu_{\perp} \sin ^{2} \gamma$.

The small peak found for normal mode at C (Fig. 3) can be attributed to the tilt of $\mathrm{FePc}$ molecules with respect to the supporting plane surface. Using the above equation for $\mu(\gamma)$ and imposing the condition that $\mu_{\|}=0$ at $\mathrm{C}$ (since the $1 \mathrm{~s} \rightarrow$ $4 \mathrm{p}_{\mathrm{z}}$ is forbidden in normal mode by symmetry), a maximum tilt angle $\phi_{T}^{\mathrm{XLPA}} \sim 12 \pm 1^{\circ}$ has been estimated. Although STM and XLPA agree in finding a non-zero $\phi_{T}$ (i.e., molecules are not fully parallel to the Au surface), quantitatively the results differ, as $\phi_{T}^{\mathrm{XLPA}} \sim 2 \phi_{T}^{\mathrm{STM}}$. At least two factors may contribute to this: $\phi_{T}^{\mathrm{XLPA}}$ is obtained from a film of about 80 molecules thick, while $\phi_{T}^{\text {STM }}$ is measured directly on the 2nd layer. Moreover, the Au substrate on the STM sample is a $\mathrm{Au}(111)$ single crystal, while the "smoothed" gold substrate is used for the thick film and XLPA experiments, even though highly ordered, ${ }^{13,15}$ it is not a single crystal, and the overall tilt may be larger.

The $a b$ initio calculations of XLPA spectra have been performed with the FDMNES code ${ }^{16,17}$ within the muffin-tin approximation for the potential. ${ }^{18}$ To simulate the film assembly keeping the inversion symmetry, FePc molecules were stacked one on top of the other, displacing the iron centers by $\pm n \mathbf{t}$, where $\mathbf{t}$ is a translation vector with coordinates $d_{x}, d_{y}, d_{z}$ and $n$-th is the layer number counted from the central molecule. The $\mathrm{H}$ atoms were not considered as they are too far from the Fe. Atomic coordinates from Ref. 1 have been used.

The translation vector is chosen in order to optimize the agreement between the calculated spectra and the experiment. Structural refinement led to the following optimized values: $d_{x}=1.48 \AA, d_{y}=0$, and $d_{z}=3.25 \AA$, furthermore in qualitative agreement with STM results $\left(d_{x}=1.0 \pm 0.3 \AA\right)$. In Fig. 3, we show the comparison between the experimental data and the theoretical spectra obtained on the optimized structure. In normal incidence, the agreement is excellent. All spectral structures in the experiment are equally retrieved by the calculation, including the shoulder at $7125 \mathrm{eV}$. The main structures at 7131 and $7141 \mathrm{eV}$ are calculated at the correct energies and relative intensities. In grazing incidence, the agreement between the calculated and the experimental spectra is very good, with the exception of the global 
intensities of the spectra structures in the energy range between 7120 and $7145 \mathrm{eV}$. Nonetheless, the feature at $7126 \mathrm{eV}$ and the high energy structure at $7160 \mathrm{eV}$ are well reproduced in terms of energy positions and intensities. Altogether this motivates the very good agreement between the theoretical and experimental XNLD.

Our combined STM and XAS work shows the presence of a tilt between the Au substrate and the plane of the FePc molecules in "lying" columnar growth of about $9 \pm 4^{\circ}$, which depends on the nature of the Au substrate. Moreover, the magnetic thick films grow in the TF phase, a different stacking from the bulk crystalline $\alpha$ or $\beta$ phase. Our results highlight the need for a detailed study of the origin of ferromagnetism in the FePc - TF phase. ${ }^{11}$

The financial support of the Spanish financial agency MINECO MAT2011-2379 and MAT2014-53921-R, Aragonese DGA-IMANA E34 (co-funded by European Social Fund), as well as European Union FEDER funds is acknowledged. The sample preparation and initial structural characterization were supported by the Office of Basic Energy Science, U.S. Department of Energy, BES-DMS funded by the Department of Energy's Office of Basic Energy Science, DMR under Grant No. DE FG03 87ER45332 and NSF DMR 0847552. X-ray data were obtained at the ESRF under experiment HE-3009. STM studies were performed at the Laboratorio de Microscopías Avanzadas INA, Universidad de Zaragoza.

${ }^{1}$ J. F. Kirner, W. Dow, and W. R. Scheidt, Inorg. Chem. 15, 1685 (1976).

${ }^{2}$ C. Ercolani, C. Neri, and P. Porta, Inorg. Chim. Acta 1, 415 (1967).

${ }^{3}$ C. W. Miller, A. Sharoni, G. Liu, C. N. Colesniuc, B. Fruhberger, and I. K. Schuller, Phys. Rev. B 72, 104113 (2005).

${ }^{4}$ H. Peisert, T. Schwieger, J. M. Auerhammer, M. Knupfer, M. S. Golden, J. Fink, P. R. Bressler, and M. Mast, J. Appl. Phys. 90, 466 (2001).

${ }^{5}$ T. Gredig, K. P. Gentry, C. N. Colesniuc, and I. K. Schuller, J. Mater. Sci. 45, 5032 (2010).

${ }^{6}$ S. Ahmadi, M. N. Shariati, S. Yu, and M. Göthelid, J. Chem. Phys. 137, 084705 (2012).

${ }^{7}$ C. Montón, I. Valmianski, and I. K. Schuller, Appl. Phys. Lett. 101, 133304 (2012).

${ }^{8}$ X. Chen, Y.-S. Fu, S.-H. Ji, T. Zhang, P. Cheng, X.-C. Ma, X.-L. Zou, W.H. Duan, J.-F. Jia, and Q.-K. Xue, Phys. Rev. Lett. 101, 197208 (2008).

${ }^{9}$ M. Evangelisti, Encyclopedia of Supramolecular Chemistry (Marcel Decker, Inc., New York, 2004), p. 1069.

${ }^{10}$ G. Filoti, M. D. Kuz'min, and J. Bartolomé, Phys. Rev. B 74, 134420 (2006).

${ }^{11}$ J. Bartolomé, F. Bartolomé, L. M. García, G. Filoti, T. Gredig, C. N. Colesniuc, I. K. Schuller, and J. C. Cezar, Phys. Rev. B 81, 195405 (2010).

${ }^{12}$ Z. H. Cheng, L. Gao, Z. T. Deng, Q. Liu, N. Jiang, X. Lin, X. B. He, S. X. Du, and H.-J. Gao, J. Phys. Chem. C 111, 2656 (2007).

${ }^{13}$ S. Yim, S. Heutz, and T. S. Jones, Phys. Rev. B 67, 165308 (2003).

${ }^{14}$ G. Liu, T. Gredig, and I. K. Schuller, EPL 83, 56001 (2008).

${ }^{15}$ T. Gredig, C. N. Colesniuc, S. A. Crooker, and I. K. Schuller, Phys. Rev. B 86, 014409 (2012).

${ }^{16}$ Y. Joly, Phys. Rev. B 63, 125120 (2001).

${ }^{17}$ O. Bunau and Y. Joly, J. Phys.: Condens. Matter 21, 345501 (2009).

${ }^{18}$ J. C. Slater, Phys. Rev. 51, 846 (1937). 